\title{
Identification of Novel Wsf1 Mutations in a Sicilian Child with Wolfram Syndrome
}

\author{
Giuseppe Pizzolanti ${ }^{1 \dagger}$, Laura Tomasello ${ }^{1 \dagger}$, Antonina Coppola $^{1}$, Maria Pitrone ${ }^{1}$, Concetta Baiamonte ${ }^{1}$, Alessandro Ciresi $^{1}$, Renata Lorini ${ }^{2}$, \\ Walter Arancio ${ }^{1}$ and Carla Giordano ${ }^{1 *}$
}

${ }^{1}$ Laboratory of Molecular Endocrinology, Di.Bi.M.I.S, University of Palermo, Italy

${ }^{2}$ Clinica Pediatrica, Istituto Giannina Gaslini, University of Genoa, Italy

tThese authors contributed equally to this article

\begin{abstract}
Wolfram Syndrome (WS) is a rare hereditary disease with autosomal recessive inheritance with incomplete penetrance. It is characterized by diabetes mellitus associated with progressive optic atrophy. The diagnosis is essentially clinical and mutation analysis is used to confirm the diagnosis. In the present study we describe the clinical and molecular features of a diabetic child carrying two novel WFS1 mutations. The Sicilian proband and his non-affected family were studied. Ophthalmologic examination included: visual acuity determination and funduscopy, optical coherent tomography, retinal fluorangiography, perimetry and electroretinogram. Molecular methods: automatic sequencing of PCR amplified WFS1 gene fragments and qRT-PCR analysis of WFS1 transcripts. 3 WSF1 mutations have been identified in the proband. One allele carries 2 paternally inherited mutations (c.1332 $C>G$ and c. $1631 \mathrm{C}>\mathrm{G}$ ) in exon-8, never annotated before, in heterozygosis with one "de novo" classic mutation (c.505 G>A) in exon-5. In addition, we report an unexpected molecular feature: higher WFS1 mRNA levels in the proband compared to the father.
\end{abstract}

Keywords: Wolfram syndrome; WFS1; DIDMOAD; Diabetes

\section{Introduction}

Wolfram syndrome (WS; OMIM 222300) was first described by Wolfram and Wagener as the association of childhood-onset Diabetes Mellitus (DM) and Optic Atrophy (OA). The disorder is also known with the acronym DI-DM-OA-D, since additional clinical features observed in the majority of patients include Diabetes Insipidus (DI) and sensori neuronal deafness at high-frequency (D). It is a progressive neurodegenerative disorder, possibly developing ataxia, peripheral neuropathy, and psychiatric illness. Non autoimmune, non HLA-linked diabetes mellitus develops in the first decade of life, at a median age of 6 years, and usually administration of insulin is required. Other complications include urogenital anomalies, and less frequent complications such as gastrointestinal anomalies, most often dysmotility and/or chronic diarrhoea. Primary gonadal atrophy is common in males, and many females have menstrual irregularity and delayed menarche [2,3]. Follow-up studies demonstrate that WS patients could develop all symptoms if they live long enough. Death occurs prematurely, with a median age of 30 years, often from respiratory failure caused by brain stem atrophy. The prevalence of WS was estimated as $1 / 770,000$ in the UK, with a carrier frequency of 1 in 354; and 1/100,000 in the North American population, based on the $1 / 175$ occurrence of optic atrophy in a juvenile-diabetes clinic.

The WFS1 gene (MIM\# 606201) maps to chromosome 4p16, and consists of eight exons, encompassing $33.4 \mathrm{~kb}$ of genomic DNA: exon 1 is noncoding, exons 2-7 are small coding exons and the largest exon, exon 8 , is $2.6 \mathrm{~kb}$ long. Most patients carry mutations in the WFS1 gene; a second form of WS is caused by a homozygous mutation in a recently identified zinc-finger protein, Endoplasmatic Reticulum Intermembrane Small protein (ERIS) encoded by the WFS2 gene, mapping to 4q22-q25 [4-7]. Defects in mitochondrial DNA were reported in some sporadic cases, but the mitochondrial genome was not found to be systematically involved in the disease [8-11]. Mutations reported in the WFS1 gene give rise to two distinct phenotypes: autosomal recessive WS and autosomal dominant low-frequency sensorineural hearing loss (AD LFSNHL) $[5,12]$.
The WFS1 gene transcribes a $3.6 \mathrm{~kb}$ mRNA that encodes Wolframin, a 890 -amino-acid protein, abundantly detected in the adult human heart, brain, placenta, lung, and pancreas beta-cells $[3,5,6]$. Wolframin is a calmodulin glycoprotein resident in the membrane of the Endoplasmic Reticulum (ER) involved in the ER stress signalling $[3,13]$. It has been speculated that in WS patients a dysregulation of the ER stress signalling could lead to cell death in the affected cells such as pancreatic beta-cells or neurons $[3,13]$.

Mutations associated with the disease phenotype are distributed along the entire gene, with homozygous and compound heterozygous genotypes. To date, over 200 distinct mutations in WFS1 have been identified in WS individuals from different ethnic backgrounds, and these include a variety of missense, nonsense, and frameshift insertion/ deletion mutations [3,5,6,13-22].

Cognitive impairment seems to be more frequent than how much estimated in the past, and epilepsy is diffuse in the children with an early onset of neurological signs, associated with malformations of cortical development with white matter involvement, including leukoencephalopathy

The majority of patients with DIDMOAD carry mutations that presumably result in a loss of function of Wolframin. This suggests that a lack of Wolframin protein is the disease-causing agent $[3,5,6,13-18]$.

*Corresponding author: C Giordano, Laboratory of Molecular Endocrinology, Section of Endocrinology, Di.Bi.M.I.S, Piazza delle Cliniche 290127 Palermo, Italy, Tel: +390916552109; Fax:+390916552123; E-mail: carla.giordano@unipa.it

Received July 25, 2014; Accepted September 23, 2014; Published September 29, 2014

Citation: Pizzolanti G, Tomasello L, Coppola A, Pitrone M, Baiamonte C, et al. (2014) Identification of Novel Wsf1 Mutations in a Sicilian Child with Wolfram Syndrome. J Genet Syndr Gene Ther 5: 245. doi:10.4172/2157-7412.1000245

Copyright: (c) 2014 Pizzolanti G, et al. This is an open-access article distributed under the terms of the Creative Commons Attribution License, which permits unrestricted use, distribution, and reproduction in any medium, provided the original author and source are credited. 
In the present study we describe the clinical and molecular features of a novel WFS1 mutation carrying child and his family living in Sicily (Italy).

\section{Methods}

\section{Patient and family history}

Our study concerns a 12-year-old male, the younger of two children, with familial history of diabetes mellitus (both grandmothers and a paternal uncle with type 2 diabetes mellitus). No family member was suffering from psychiatric symptoms, visual disorders or hearing loss. The parents were healthy and non-consanguineous. All family members showed no signs of WS. The proband was diagnosed as affected by insulin-dependent diabetes mellitus without anti-Islet Cell Antibodies (ICA), antiGAD65 and Insulin-Autoantibodies (IAA), when he was 3 years old, with onset symptoms characterized by polyuria, polydipsia and ketosis. From the moment of diagnosis he was treated with basal-bolus insulin therapy, leading to poor control of his glycemic levels. He complained of vision decrease when he was 7 years old. Only at the age of 11 a visual examination was performed, including visual acuity determination and funduscopy. At examination, his visual acuity was $4 / 10$ bilaterally and remained constant in subsequent years. Hypopigmentation with a waxy pallor of the optic disc was found in the macular area, so optical coherence tomography (OCT-Copernicus) was performed a few months later. The right and left optic nerve heads showed a cup-to-disc ratio of 0.557 and 0.551 , respectively. A thickness reduction of the ganglion nerve fibres (mean values of 81 and $74 \mathrm{~m}$ in the right and left eye, respectively) and a reduced optic disc size (1.272 and $1.506 \mathrm{~mm}^{2}$ in the right and left eye, respectively) were found. Some microhemorrhages in the macular area were also described. Retinal Fluorangiography (FAG) was normal in both eyes, with normal perimetry and Pattern Electroretinogram (PERG) amplitude. He showed a normal age-related, pubertal development assessed by the Tanner criteria (PH3G2) and a regular growth velocity. Dental examination only showed teeth discoloration. No gastrointestinal, psychiatric or neurological disorders and abnormalities of hearing function were reported by the parents. On the basis of the ocular characteristics WS was diagnosed.

\section{DNA analysis}

Genomic DNA from peripheral blood of the proband and his family members was extracted using a commercially available kit (Qiagen, Hilden, Germany).

The 2-8 exons of WFS1 were amplified by PCR using sets of primers and conditions as previously published by Strom et al . PCR products were analyzed in agarose gels and subsequently amplified templates were purified with AMPure magnetic beads (Agencourt, BeckmanCoulter) according to the manufacturer's instructions and an aliquot of purified PCR product was cycle sequenced by the BigDye Terminator v3.1 Cycle Sequencing Kit (Applied Biosystems). These reactions were further processed by running a clean-up procedure with CleanSEQ beads (Agencourt, Beckman-Coulter) on a Biomek 3000 Laboratory automated workstation (Beckman-Coulter). Finally, the products were sequenced on a 3130XL Genetic Analyzer (Applied Biosystems). Wildtype and mutated WFS1 sequences were compared manually.

\section{RNA analysis}

Total RNA samples were extracted by Ficoll-separated lymphocytes from the proband and his father along with a pool of lymphocytes from a controlgroupmatchedforageandsex, using the RNeasyMiniKit(Qiagen,
Milan, Italy) following manifacturer's instructions. A neuroblastoma cell line (LAN5) used as a positive control. WFS1 expression was analysed by real-time quantitative RT-PCR (qRT-PCR) in individual samples and WFS1 mRNA levels were quantified in comparison to $\beta$-actin mRNA expression. WFS1 PCR primers were purchased from Quiagen (Quantitect Primer Hs_WFS1_1_SG QT00082663) the amplicon size was 117 bp (Suppl. Figure 1); $\beta$-actin primers were obtained from RealTimePrimers.com (Elkins Park, PA, USA) with the following sequences: F 5'-GGACTTCGAGCAAGAGATGG-3'; R 5'-AGCACTGTGTTGGCGTACAG-3'; the amplicon size was 234 bp. All reactions were performed using the LightCycler 1.0 (Roche Diagnostics GMbH, Germany). Briefly, 2 g of total RNA was retrotranscribed using the ImProm- $\mathrm{II}^{\mathrm{ma}}$ Reverse Transcription System (Promega, Milan, Italy). Each RNA was combined with 11 of oligo16dT (Promega Milan, Italy) and thermally denatured at $70^{\circ} \mathrm{C}$ for 5 minutes and then chilled on ice. As a final step, the template-oligo$\mathrm{dT}$ combination was added to the reaction mix (4 1 ImProm- $-\mathrm{II}^{\mathrm{sx}} 1 \mathrm{X}$ Reaction Buffer, 11 ImProm-II ${ }^{\mathrm{Tx}}$ Reverse Transcriptase, 3.2 1 Magnesium Chloride $3 \mathrm{mM}, 1 \mathrm{ldNTPs}$ mix $10 \mathrm{mM}$ and 11 Recombinant RNasin Ribonuclease Inhibitor $1 \mathrm{u} / \mathrm{l}$ ) and nuclease-free water was added to 20 1 of final volume on ice. Following an initial annealing at $25^{\circ} \mathrm{C}$ for 5 minutes, the reaction was incubated at $42^{\circ} \mathrm{C}$ for one hour. In order to heat-inactivate the ImProm-II Reverse Transcriptase the reaction mix was finally incubated at $70^{\circ} \mathrm{C}$ for 15 minutes.

Real-time PCR was performed using 21 of cDNA diluited 1:5, plus 101 of Quantitech master mix (Qiagen), 21 of Quantitect Primer Hs_WFS1_1_SG or $\beta$-actin primers, and nuclease-free water to 201 of final volume. A standard reference curve was performed in parallel using RNAs extracted from LAN5 cell line serially 1:2 diluited starting from $100 \mathrm{ng}$ reaction to $3.125 \mathrm{ng}$.

The thermal profile consisted of an initial activation step at $95^{\circ} \mathrm{C}$ for 15 minutes, followed by 45 cycles of $95^{\circ} \mathrm{C}$ for 10 seconds, $60^{\circ} \mathrm{C}$ for 15 seconds, $72^{\circ} \mathrm{C}$ for 15 seconds. Fluorescence in the FL1 channel was measured once per cycle at the end of the $72^{\circ} \mathrm{C}$ step. At the end of PCR cyccling, a standard melting curve analysis was performed; briefly the amplicons were heated from $55^{\circ} \mathrm{C}$ to $95^{\circ} \mathrm{C}$ at $0.1^{\circ} \mathrm{C} / \mathrm{min}$ with continuous fluorescence acquisition and as expected a single peak per amplicon was detected. Data were analysed using the relative quantification method of Pfaffl with efficency correction, using $\beta$-actin as normalization gene.

\section{Results}

Mutational screening of the WFS1 gene (Table 1A, Figure 1B) revealed in the affected proband a "de novo" missense mutation in exon 5 (c. $505 \mathrm{G}>\mathrm{A}$ ) in heterozygous with an allele carrying 2 variations in exon 8: c.1332 $\mathrm{C}>\mathrm{G}$ and c.1631C $>\mathrm{G}$, both paternally inherited and never annotated before. c. $1332 \mathrm{C}>\mathrm{G}$ causes a premature STOP codon introduction at position 444 and c. $1631 \mathrm{C}>\mathrm{G}$ causes a substitution from a serine to a cysteine at position 544 . The novel mutations were present both in the proband and his father; no mutation-caused disease was found in the other family members analysed (Figure 1A), strongly suggesting the paternal inheritance of c.1332 $C>G$ and c.1631C $>\mathrm{G}$ mutations from a single allele and the "de novo" acquisition of the c.505 $\mathrm{G}>\mathrm{A}$ in the other allele of the proband. Many neutral polymorphisms were also detected in the proband (Table 1B).

As regards WFS1 expression, we found higher mRNA levels in the WS proband than in healthy subjects ( 5 children of the same age). The levels of WFS1 mRNA were also increased in the father (Figure 1C), 
A

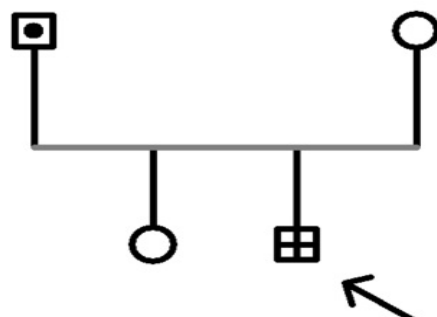

C

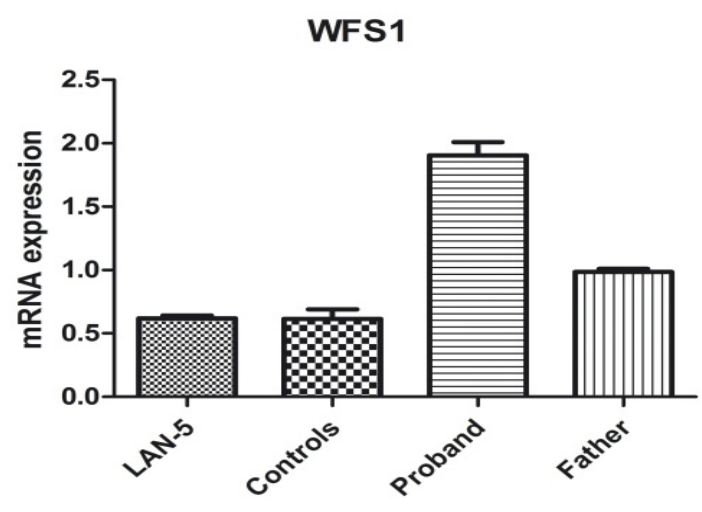

\section{B}

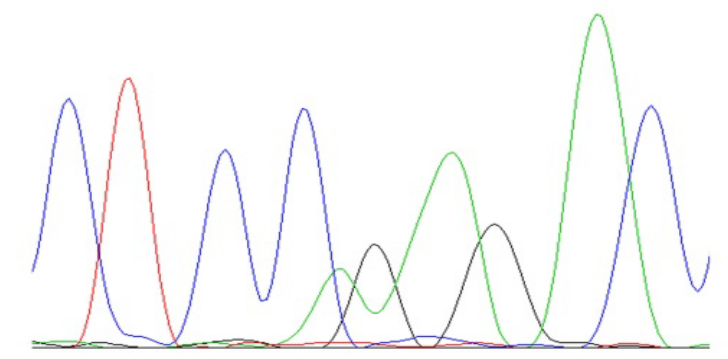

Ex.5

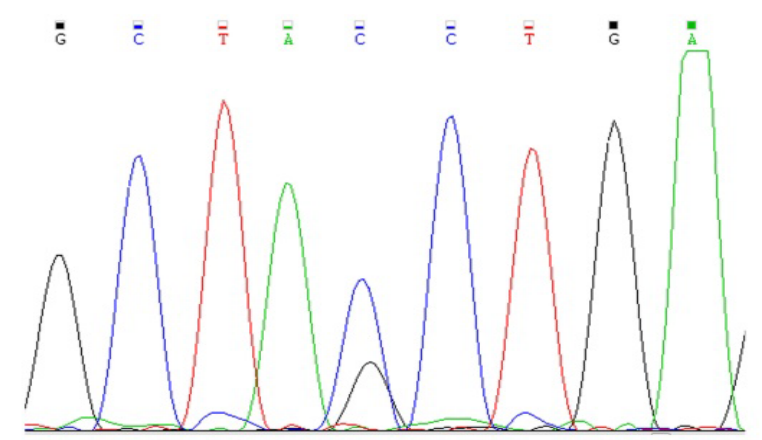

Ex.8

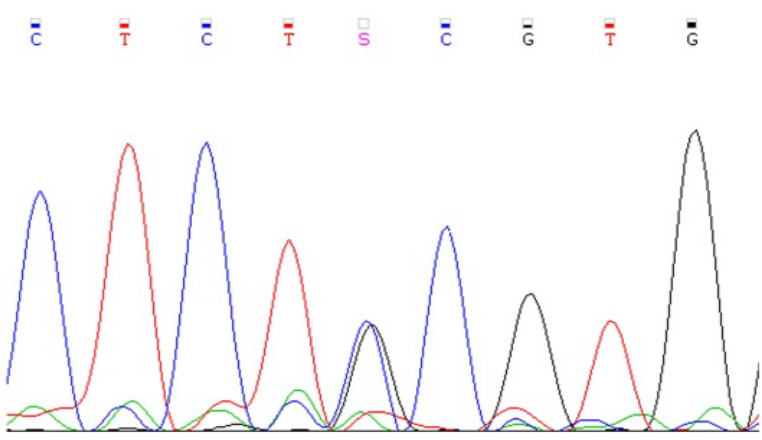

Ex.8

Figure 1: A) The pedigree of family. The arrow points to the proband. B) WFS1 gene novel mutations in proband. Representative electropherograms from the affected patient show heterozygous mutations in exon $5(505 \mathrm{G}>\mathrm{A})$ and in exon $8(1332 \mathrm{C}>\mathrm{G} ; 1631 \mathrm{C}>\mathrm{G})$. C) qRT-PCR of WFS1 mRNA normalized expression against beta-actin in:

Column 1: Positive control LAN-5 cell line;

Column 2: Healthy subjects (mean values of 5 children);

Column 3: Proband;

Column 4: Proband's father.

even if to a less degree, who is only an unaffected heterozygous mutant without evident WS phenotype.

\section{Discussion}

Genetic analyses in WS patients have identified a wide spectrum of mutations: many of them showed loss-of-function mutations such as stop, frame-shift and splice site mutations while missense mutations were detected in $35 \%$ of the cases. Different types of mutations located in the hydrophilic C-terminal part of the protein lead to complete and severe WS underlining the functional importance of Wolframin C-terminus [5,14,24-26]. 


\begin{tabular}{|c|c|c|c|}
\hline Exon & Nucleotide change & Type of mutation & Amminoacid change \\
\hline 5 & c. $505 \mathrm{G}>\mathrm{A}$ & Missense & p.E169K \\
\hline 8 & c.1332C>G & Nonsense & p.Y444X \\
\hline 8 & c.1631C>G & Missense & p.S544C \\
\hline
\end{tabular}

Table 1A: Missense and Nonsense Mutations in Proband's WFS1.

\begin{tabular}{|c|c|c|c|c|c|}
\hline Exon & Polymorphism RefSNP accession ID & Nucleotide change & Type of Polymorphism & Amminoacid change & State \\
\hline 8 & rs1801206CT & c.1185T>C & Synonymous & V395V \\
\hline 8 & rs1801208GA & c.1367G $>$ A & Non-Synonymous & R456H \\
\hline 8 & rs1801214TT & c.1500T>C & Synonymous & H500N \\
\hline 8 & rs734312GA & c.1998A $>$ G & Non-Synonymous & Homozygous \\
\hline 8 & rs1046314AA & c.2433G $>$ A & Synonymous & K811K \\
\hline 8 & rs1046316AG & c.2565G $>$ A & Synonymous & Homozygous \\
\hline
\end{tabular}

Table 1B: Polymorphisms in Proband's WFS1.

The diagnosis of Wolfram Syndrome is essentially clinical and mutation analysis is usually used to confirm diagnosis, though a recent update of WFS1 mutations revealed no obvious hot spots or clustering and mutations appeared to be distributed randomly within the entire coding sequence of the gene. The growing number of WS reports confirmed the wide spectrum of WFS1 mutations and the variations in phenotypes, fostering better understanding of the genotypephenotype correlation. Recent result highlighted the difficulties in a clear definition of the inclusion criteria; briefly WSF1 mutations have been clustered in three classes: type I, which lead to complete depletion of WFS1 protein due to the activation of nonsense-mediated decay, type II, which lead to complete degradation of WFS1 protein by keeping functional the degron in WFSland type III, leading to the expression of a defective or shorter WFS1 protein. In some cases, optic atrophy and diabetes mellitus might not be the early manifestations of the clinical conditions in WS, and the age of onset and the disease progression might be correlated to the genotype of the affected individual; moreover, genotypic prevalence seems to exist in different geographical areas. It seems clear that the decline of $\beta$-cells population and activity is generally an early event in WS patients and that glucose toxicity enhances the ER stress-mediated progression of the disease.

In our study we describe the clinical and genetic features of a child with WS caused by a compound heterozygous mutations: a "de novo" missense mutation in exon 5 (previously reported as a causative mutation in a WFS family) in an allele and two novel not annotated mutations (c.1332 C>G; p.Y444X and c.1631 C>G; p.S544C) in exon 8 . The two novel heterozygous changes in exon 8 were found in the proband and his father, pointing to the paternal inheritance of the mutations. Instead, the missense change E169K, was acquired sporadically in the proband, leading to the compound heterozygosis of the WFS1 gene and thus to the WF. The absence of clinical signs of the syndrome in the father and the severe WF phenotype in the child confirm these data. The missense mutation at exon 5 replaces a glutamic acid residue at position 169 with a lysine residue in the Wolframin extra reticular domain, while the paternally inherited nonsense mutation in exon 8 introduces a premature STOP codon at position 444 that disrupts part of the transmembrane domain.

The Wolframin protein is expressed in many organs with the highest levels in the central nervous system, pancreas beta-cells, heart and muscle followed by the liver and lower levels in the kidney and spleen. However, in WS patients only selected populations of neuronal and endocrine cells are affected, probably the most affected by the impaired function of Wolframin $[3,5,6,13,15]$. The most probable mechanism is a hyper-activation of the ER stress system that leads in turn to cell death in susceptible cells.
Interestingly, we found an unexpected higher mRNA levels in both the proband and to a less degree in his father than in healthy control subjects, although we were not able to detect Wolframin protein in our samples (data not shown). These data suggest that the lack of Wolframin activity could be due to the great instability of the mutants proteins instead of an increased turnover of the aberrant transcripts by the nonsense-mediated mRNA decay, as suggested by the classification reported in the genotypic classification of patients with Wolfram syndrome. Moreover, we can hazard that in the peripheral leukocytes of the proband, at least, a positive feedback mechanism increasing the WFS1 gene transcription could exist to compensate for the Wolframin deficit.

In conclusions, our findings increase the spectrum of WFS1 mutations in WS and reinforce the hypothesis of the existence of different WS phenotypes related to the different WFS1 mutations. Accordingly to the recent systematic review on the genotypic classification of patients with Wolfram syndrome , the "de novo" missense mutation isolated in exon 5 could be considered as a type II mutation which lead to complete degradation of WFS1 protein, while the mutations on exon 8 could be considered a type III mutation, leading to the expression of a shorter, readily degraded WFS1 protein.

\section{Acknowledgments}

We thank the patient and his family for their participation in this study. This study was partially support by "Progetto d'Ateneo 2006 " to C.G. and G.P.

\section{References}

1. Wolfram DJ, Wagener HP (1938) Diabetes mellitus and simple optic atrophy among siblings: report of four cases. Mayo ClinProc 13:715-718

2. Minton JA, Rainbow LA, Ricketts C, Barrett TG (2003) Wolfram syndrome. Rev EndocrMetabDisord 4: 53-59.

3. Rigoli L, Lombardo F, Di Bella C (2011) Wolfram syndrome and WFS1 gene. Clin Genet 79: 103-117.

4. Barrett TG, Bundey SE, Macleod AF (1995) Neurodegeneration and diabetes: UK nationwide study of Wolfram (DIDMOAD) syndrome. Lancet 346: 14581463.

5. Inoue H, Tanizawa Y, Wasson J, Behn P, Kalidas K, et al. (1998) A gene encoding a transmembrane protein is mutated in patients with diabetes mellitus and optic atrophy (Wolfram syndrome). Nat Genet 20: 143-148.

6. Strom TM, Hortnagel K, Hofmann S, et al. (1998) Diabetes insipidus, diabetes mellitus, optic atrophy and deafness (DIDMOAD) caused by mutations in a novel gene (wolframin), coding for a predicted transmembrane protein. Hum Mol Genet. 7:2021-2028

7. Amr S, Heisey C, Zhang M, Xia XJ, Shows KH, et al. (2007) A homozygous mutation in a novel zinc-finger protein, ERIS, is responsible for Wolfram syndrome 2. Am J Hum Genet 81: 673-683.

8. Rötig A, Cormier V, Chatelain P, Francois R, Saudubray JM, et al. (1993) 
Citation: Pizzolanti G, Tomasello L, Coppola A, Pitrone M, Baiamonte C, et al. (2014) Identification of Novel Wsf1 Mutations in a Sicilian Child with Wolfram Syndrome. J Genet Syndr Gene Ther 5: 245. doi:10.4172/2157-7412.1000245

Page 5 of 5

Deletion of mitochondrial DNA in a case of early-onset diabetes mellitus, optic atrophy, and deafness (Wolfram syndrome, MIM 222300). J Clin Invest 91 1095-1098.

9. Barrientos A, Casademont J, Saiz A, Cardellach F, Volpini V, et al. (1996) Autosomal recessive Wolfram syndrome associated with an 8.5-kb mtDNA single deletion. Am J Hum Genet 58: 963-970

10. Jackson MJ, Bindoff LA, Weber K, Wilson JN, Ince P, et al. (1994) Biochemica and molecular studies of mitochondrial function in diabetes insipidus, diabetes mellitus, optic atrophy, and deafness. Diabetes Care 17: 728-733.

11. Barrett TG, Scott-Brown M, Seller A, Bednarz A, Poulton K, et al. (2000) The mitochondrial genome in Wolfram syndrome. J Med Genet 37: 463-466.

12. Young TL, Ives E, Lynch E, Person R, Snook S, et al. (2001) Non-syndromic progressive hearing loss DFNA38 is caused by heterozygous missense mutation in the Wolfram syndrome gene WFS1. Hum Mol Genet 10: 25092514.

13. Boutzios G, Livadas S, Marinakis E, Opie N, Economou F, et al. (2011) Endocrine and metabolic aspects of the Wolfram syndrome. Endocrine 40: $10-13$.

14. de Heredia ML, Clèries R, Nunes V (2013) Genotypic classification of patients with Wolfram syndrome: insights into the natural history of the disease and correlation with phenotype. Genet Med 15: 497-506.

15. Rohayem J, Ehlers C, Wiedemann B, Holl R, Oexle K, et al. (2011) Diabetes and neurodegeneration in Wolfram syndrome: a multicenter study of phenotype and genotype. Diabetes Care 34: 1503-1510.

16. Chaussenot A, Bannwarth S, Rouzier C, Vialettes B, Mkadem SA, et al. (2011) Neurologic features and genotype-phenotype correlation in Wolfram syndrome. Ann Neurol 69: 501-508.
17. Chaussenot A, Rouzier C, Quere M, Plutino M, Ait-El-Mkadem S, et al. (2014) Mutation update and uncommon phenotypes in a French cohort of 96 patients with WFS1-related disorders. ClinGenet

18. Hardy C, Khanim F, Torres R, Scott-Brown M, Seller A, et al. (1999) Clinical and molecular genetic analysis of 19 Wolfram syndrome kindreds demonstrating a wide spectrum of mutations in WFS1. Am J Hum Genet 65: 1279-1290.

19. vanvenOuweland JM, Cryns K, Pennings RJ, Walraven I, Janssen GM, et al. (2003) Molecular characterization of WFS1 in patients with Wolfram syndrome. J MolDiagn 5: 88-95.

20. Khanim F, Kirk J, Latif F, Barrett TG (2001) WFS1/wolframin mutations, Wolfram syndrome, and associated diseases. Hum Mutat 17: 357-367.

21. Giuliano F, Bannwarth S, Monnot S, Cano A, Chabrol B, et al. (2005) Wolfram syndrome in French population: characterization of novel mutations and polymorphisms in the WFS1 gene. Hum Mutat 25: 99-100.

22. d'Annunzio G, Minuto N, D'Amato E, de Toni T, Lombardo F, et al. (2008) Wolfram syndrome (diabetes insipidus, diabetes, optic atrophy, and deafness) clinical and genetic study. Diabetes Care 31: 1743-1745.

23. Pfaffl MW (2001) A new mathematical model for relative quantification in realtime RT-PCR. Nucleic Acids Res 29: e45.

24. Barrett TG, Bundey SE (1997) Wolfram (DIDMOAD) syndrome. J Med Genet 34: 838-841.

25. Sam W, Qin H, Crawford B, Yue D, Yu S (2001) Homozygosity for a 4-bp deletion in a patient with Wolfram syndrome suggesting possible phenotype and genotype correlation. Clin Genet 59: 136-138.

26. Hofmann S, Philbrook C, Gerbitz KD, Bauer MF (2003) Wolfram syndrome: structural and functional analyses of mutant and wild-type wolframin, the WFS 1 gene product. Hum Mol Genet 12: 2003-2012.
Citation: Pizzolanti G, Tomasello L, Coppola A, Pitrone M, Baiamonte C, et al. (2014) Identification of Novel Wsf1 Mutations in a Sicilian Child with Wolfram Syndrome. J Genet Syndr Gene Ther 5: 245. doi:10.4172/2157-7412.1000245
Submit your next manuscript and get advantages of OMICS Group submissions

Unique features:

- User friendly/feasible website-translation of your paper to 50 world's leading languages

- Audio Version of published paper

- Digital articles to share and explore

Special features:

- 350 Open Access Journals

30,000 editorial team

21 days rapid review process

Quality and quick editorial, review and publication processing

Indexing at PubMed (partial), Scopus, EBSCO, Index Copernicus and Google Scholar etc

Sharing Option: Social Networking Enabled

Authors, Reviewers and Editors rewarded with online Scientific Credits

Better discount for your subsequent articles

Submit your manuscript at: http://www.editorialmanager.com/omicsgroup/ 